\title{
Dois teoremas sôbre a função gama
}

\author{
FREDERICO PIMENTEL GOMES
}

Escola Superior de Agricultura "Luiz de Queiroz" Universidade de S. Paulo - Piracicaba

\section{INDICE}

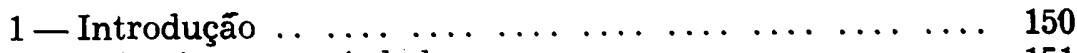

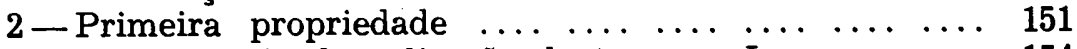

3 - Um exemplo de aplicação do teorema I ... . . . ... 154

4 -Segunda propriedade $\ldots \ldots \ldots \ldots \ldots \ldots \ldots \ldots$

5 - Um exemplo de aplicação do teorema II ... . . . ... 156

$\begin{array}{llllllllll}6 \text {-Abstract } \ldots \ldots & \ldots & \ldots & \ldots & \ldots & \ldots & \ldots & \ldots & \ldots & \ldots\end{array}$

$\begin{array}{llllllllll}7 \text { - Bibliografia } & \ldots & \ldots & \ldots & \ldots & \ldots & \ldots & \ldots & \ldots & \ldots\end{array}$ 


\section{INTRODUÇÃO}

Sendo $u$ um número real ou complexo cuja parte real $\mathrm{R}(\mathrm{u})$ é maior que -1 , podemos definir a função gama pela identidade :

$$
\Gamma(u+1)=\int_{0}^{\infty} t^{u} e^{-t} d t,
$$

Uma propriedade importante da função gama, que tem aplicação na teoria das funções características do Cálculo de Probabilidades, é que na integral acima podemos substituir a variável real $t$ por uma variável complexa.

$$
\mathrm{z}=\mathrm{x}+\mathrm{yi}=\varrho(\cos \varphi+\mathrm{i} \operatorname{sen} \varphi),
$$

desde que tenhamos

$$
-\frac{\pi}{2}<q<\frac{\pi}{2},
$$

sem que isto altere o valor da integral.

Se em (1.1.) fizermos $t=w^{2}$, com $w \supseteq 0$, obteremos

$$
\text { (1.2) } \Gamma(u+1)=2 \int_{0}^{\infty} w^{2 u+1} e^{-w^{2}} d w \text {. }
$$

e se $2 u+1$ fôr um número par não negativo, a função

$$
w^{2 u}+1 e-w^{2}
$$

será par, de sorte que teremos

$$
\Gamma(u+1)=\int_{-\infty}^{\infty} w^{2 u+1} e^{-w^{2}} d w
$$

Nesta integral podemos substituir $w$ por $z=w+f i$, onde $f$ é constante, também sem alterar o valor da integral. 
Estas duas propriedades são implicitamente admitidas por diversos autores, como KENDALL (2, p. 95), e PIMENTEL GOMES (4, p. 645) e outros chegam a sugerir o método de demonstração, como fazem CRAMER (1, p. 126) e LEVY (3, p. 178), mas não conseguimos encontrar demonstrações publicadas para elas. Daí a razão da elaboração dêste trabalho.

\section{PRIMEIRA PROPRIEDADE}

Pode ser expressa pelo teorema seguinte.

Teorema I A integral

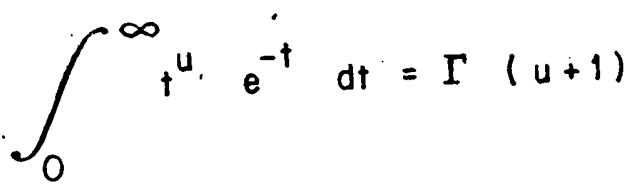

não se altera quando substituímos a variável real $t$ por uma variável complexa

$$
\mathrm{z}=\varrho(\cos \varphi+\mathrm{i} \operatorname{sen} \varphi),
$$

onde a constante $\varphi$ é tal que tenhamos

$$
-\frac{\pi}{2}<r<\frac{\pi}{2} .
$$

\section{Demonstração}

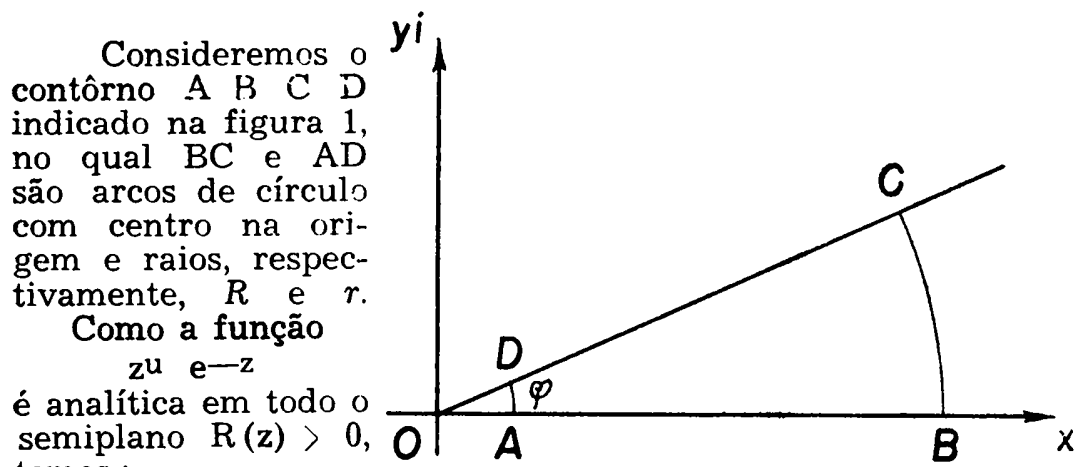
temos :

Fig. 1 


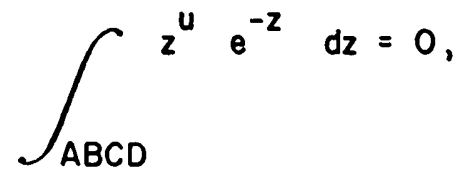

(2.1)

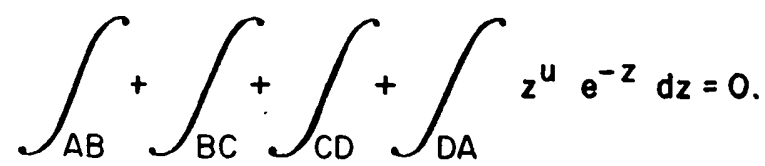

Sendo $f(z) \leq M$ ao longo de um arco regular $L$ de comprimento $c$, sabemos que

$$
\int f(z) d z \leq M c
$$

Ao longo de $\mathrm{B} \mathrm{C}$ temos $\mathrm{z}=\mathrm{R}(\cos \alpha+\mathrm{i} \operatorname{sen} \alpha), \operatorname{com} \alpha$ entre zero e $\phi$, logo

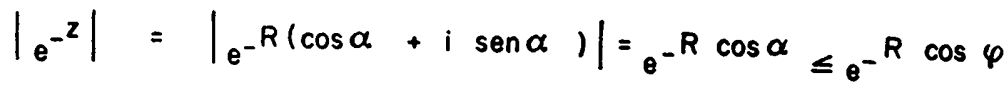

$$
\begin{aligned}
& E \text {, sendo } u=a+b i \text {, temos aindo } \\
& |z u|=\left|e^{u} \log 2\right| \\
& =\left|e^{(a+b i)(\log R+l \alpha)}\right| \\
& =e^{0} \log R-b a \\
& <K R^{0} \text {. }
\end{aligned}
$$

onde $\mathrm{K}$ é uma constante conveniente. 


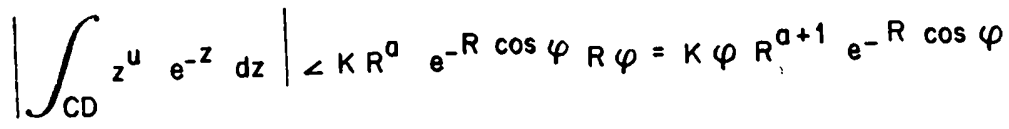

$$
\begin{aligned}
& \text { fica claro que para }-\frac{\pi}{2}<\varphi<\frac{\pi}{2} \text { temos } \\
& \lim _{R \rightarrow \infty} \int_{C D}^{u} e^{-z} d z=0
\end{aligned}
$$

Anàlogamente obtemos

$$
\int_{D A} z^{u} e^{-z} d z<k \varphi r^{a+1} e^{-r} \cos \varphi
$$

e, como $a+1>0$ por hipótese, é evidente que

$$
\lim _{r \rightarrow 0} \int_{D A}^{u} z^{-z} d z=0
$$

\section{Por definiçő}

$$
\lim _{\substack{R \rightarrow \infty \\ r \rightarrow 0}} \int_{A B} z^{u} a^{-z} d z=\int_{0}^{\infty} t^{-t} d t=\Gamma(u+1) .
$$

logo de (2.1) resulta que

$$
\text { (2.2) } \Gamma(u+1)=\lim _{\substack{R \rightarrow \infty \\ r \rightarrow 0}} \int_{A C} u e^{-z} d z=\int_{0}^{z^{u}} e^{-z} d z \text {. }
$$

sendo nesta integral 


$$
\mathrm{z}=\varrho(\cos \psi+\mathrm{i} \operatorname{sen} \psi) . \quad \text { (C.Q.D.) }
$$

Se tomarmos $\beta=\cos \phi+$ i sen $\gamma, \theta=\mathrm{t},(2.2)$ nos dará :,

$(2: 3) \quad \Gamma(\mathrm{u}+1)=i^{\mathrm{u}+1} \int_{0}^{\infty} \mathrm{t}^{\mathrm{u}} \mathrm{e}^{-\beta \mathrm{t}} \mathrm{dt}$,

onde se exige $R(\beta)>0$. Esie modo de apresentar o teorema I é dado por CRAMER (1, p. 126), sem demonstração.

3. UM EXEMPLO DE APLICAÇĀO DO TEOREMA I função

A distribuição de $x^{2}$ tem por função de frequência a

$$
\mathrm{p}(\mathrm{v})=\mathrm{C} \mathrm{v}^{\frac{\mathrm{n}}{2}-1} \quad \mathrm{e}^{-\frac{\mathrm{v}}{2}}
$$

onde $\mathrm{v}=x^{2}>0, n$ é o grau de liberdade do $x^{2} \mathrm{e}$

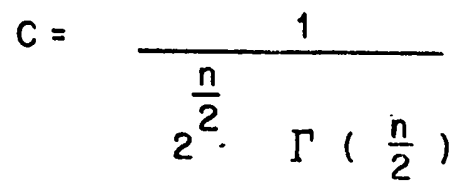

A função característica $(4$, p. 643) dessa distribuição será

$$
\begin{aligned}
\Psi(t) & =c \int_{0}^{\infty} v^{\frac{n}{2}-1} e^{-\frac{v}{2}} e^{i t v} d v \\
& =c \int_{0}^{\infty} v^{\frac{n}{2}-1} e^{-v\left(\frac{1}{2}-i t\right)} d v
\end{aligned}
$$

Tomemos

$$
z=v\left(\frac{1}{2}-i t\right), d z=\left(\frac{1}{2}-i t\right) d t
$$


e o teorema acima nos permite concluir que

$$
\begin{aligned}
\Phi(t) & =\frac{c}{\left(\frac{1}{2}-i t\right)^{\frac{n}{2}}}-\int_{0}^{\frac{n}{2}-1} z^{-z} d z \\
& =\frac{c}{\left(\frac{1}{2}-i t\right)^{\frac{n}{2}}-\Gamma\left(\frac{n}{2}\right)} \\
& =\frac{1}{(1-2 i t)^{\frac{n}{2}}}
\end{aligned}
$$

4. SEGUNDA PROPRIEDADE

E' expressa pelo teorema seguinte:

Teorema II - A igualdade

$$
\text { (4.1) } \quad \Gamma(u+1)=\int_{-\infty}^{\infty} w^{2 u+1} e^{-w^{2}} d w \text {, }
$$

válida quando $2 u+1$ é um número par não negativo, não se altera quando se substitui a variável real $\mathrm{w}$ pela variável complexa $z=w+f i$, onde $f$ é constante.

\section{Demonstração}

Consideremos $\mathrm{O}$ contôrno A B C D da figura 2. A função $z^{2 u}+1 e-z^{2}$ com $2 u+1$ número par não negativo, é analítica em todo o plano complexo. Logo :

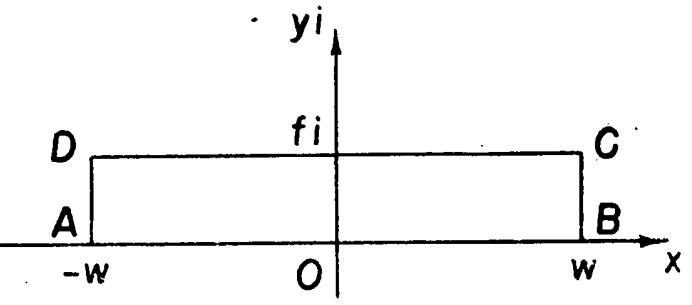

Fig. 2 
(4.2) $\int_{A B}+\int_{B C}+\int_{C D}+\int_{D A} z^{2 u+1} e^{-z^{2}} d z=0$

Sobre BC temos

$$
\begin{aligned}
&\left|e^{-z^{2}}\right|=\left|e^{-(w+y i)^{2}}\right|=e^{-w^{2}+y^{2}} \leq e^{-w^{2}+f^{2}} \\
&\left|z^{2 u+1}\right|=|z| 2 u+1=|w+f i|^{2 u+1} \leq(w+f)^{2 u+1 .}
\end{aligned}
$$

logo

$\left|\int_{B C}^{C} 2 u+1 \quad e^{-z^{2}} d z\right| \leq(w+f)^{2 u+1} e^{-w^{2}+f^{2}} f$. de onde se conclui imediatamente que

$\lim _{u \rightarrow \infty} \int_{B C}^{2 u+1} e^{-z^{2}} d z=0$.

Anàlogamente se demonstra que

$$
\lim _{w \rightarrow \infty} \int_{D A}^{2 u+1} e^{-z^{2}} d z=0
$$

logo de (4.2) e (1.3) resulta que

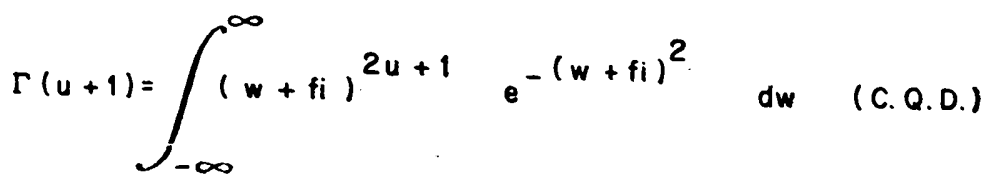

5. UM EXEMPLO DE APLICAÇÃO DO TEOREMA II

A função característica $\Phi(t)$ da distribuição normal com média zero e variância $\sigma{ }^{2}$ é $(4, \mathrm{pp} .644-645)$ : 


$$
\begin{aligned}
\Phi(t) & =\frac{1}{\sigma \sqrt{2 \pi}} \int_{-\infty}^{\infty} e^{i t x} e^{-\frac{x^{2}}{2 \sigma^{2}}} d x \\
& =\frac{e^{-\frac{1}{2} t^{2} \sigma^{2}}}{\sigma \sqrt{2 \pi}} \int_{-\infty}^{\infty} e^{-\frac{1}{2 \sigma^{2}}(x-i t \sigma)^{2}} d x
\end{aligned}
$$

Pelo teorema II temos:

$$
\begin{aligned}
& \Phi(t)=\frac{e^{-\frac{1}{2} t^{2} \sigma^{2}}}{\sigma \sqrt{2 \pi}} \int_{-\infty}^{\infty} e^{-\frac{1}{2 \sigma^{2}} x^{2}} d x \\
& =\frac{e^{-\frac{1}{2} t^{2} \sigma^{2}}}{\sigma \sqrt{2 \pi}} \sigma \sqrt{2} \quad \Gamma\left(\frac{1}{2}\right) \\
& =e^{-\frac{1}{2} t^{2} \sigma^{2}}
\end{aligned}
$$

\section{ABSTRACT}

This paper proves the following theorems on the gamma function :

\section{Theorem I}

The integral

$$
\int_{0}^{\infty} t^{u} e^{-t} d t=\Gamma(u+1),
$$


where $u$, real or complex, is such that $R(u)>-1$, will not change its value if we substitute

$$
\mathrm{z}=\varrho(\cos \varphi+\mathrm{i} \operatorname{sen} \varphi)
$$

for the real variable $t$, being $q$ constant and such that

$$
-\frac{\pi}{2}<\varphi<\frac{\pi}{2} \text {, }
$$

Theorem II

The integral

$$
\int_{-\infty}^{\infty} w^{2 u+1} e^{-w^{2}} d w=\Gamma(u+1)
$$

where $2 u+1$ is supposed to be a non negative even integer, will not change its value if we substitute $z=w+f i, f$ being a real constant, for the real variable w.

The proof of both theorems is obtained by means of the well known Cauchy theorem on contour integrals on the complex plane, as suggested by CRAMER $(1$, p. 126) and LEVY (3, p. 178).

\section{BIBLIOGRAFIA}

(1) CRAMER, Harold - Mathematical Methods of Statistics, 1a. edição. Princeton University Press, Princeton, 1946.

(2) KENDALL, Maurice C. - The Advanced Theory of Statistics, vol. I, 3a. edição. Charles Griffin, Londres, 1947.

(3) LEVY, Paul - Calcul des Probabilités, 1a. edição. Gauthier - Villars, Paris, 1925.

(4) PIMENTEL GOMES, Frederico - A Esperança Matemática e Algumas das suas Aplicações à Teoria da Amostragem. An. Esc. Sup. Agr. "Luiz de Queiroz", vol. 8, pp. 638-648, 1951. 